\title{
Management of a parturient with acute respiratory distress for caesarean delivery using spinal anaesthesia supported with novel respiratory support measures (CPAP, HFNC)
}

\author{
Leong M., Kamaraj K., Kalidindi R \\ Medway Maritime Hospital. Gillingham, Kent. \\ United Kingdom
}

\section{Background}

Pre-eclampsia and respiratory diseases are significant contributory factors for maternal cardiac arrest ${ }^{(1)}$. We report the management of a parturient undergoing emergency caesarean section with acute respiratory distress secondary to both pre-eclampsia and influenza.

\section{Case report}

- 18year- old prima gravida of $38+5 / 40$ gestation

- 3 day $\mathrm{H} / \mathrm{o}$ cough and feeling unwell which was managed with HDU care and sepsis bundle.

o Following clinical improvement labour was induced.

- Labour analgesia was provided with remifentanil PCA.

- In the following hours she became tachypnoea, tachycardia, acidotic with dropping saturation. (RR 22, HR 127, Spo2 91\%).

- Caesarean section under spinal anaesthesia supplemented with CPAP.

\section{Table.1 Investigations}

CRF

97.4

PCR

\section{8 ng}

Albumin

33

Urine Protein

$++$

$\mathrm{PH}$

7.31

$\mathrm{PCO} 2$

4.1

Po2

9

$\mathrm{BE}$

$-9.6$

17.4

$\mathrm{HCO} 3$

\section{References}

1) MBRRACE-UK Report December 2016.

2) Clinical Anaesthesiology, Sept 13, 2016:Rare but Devastating: Study Examines Maternal Cardiac Arrest Rates.

\section{Chest X ray}

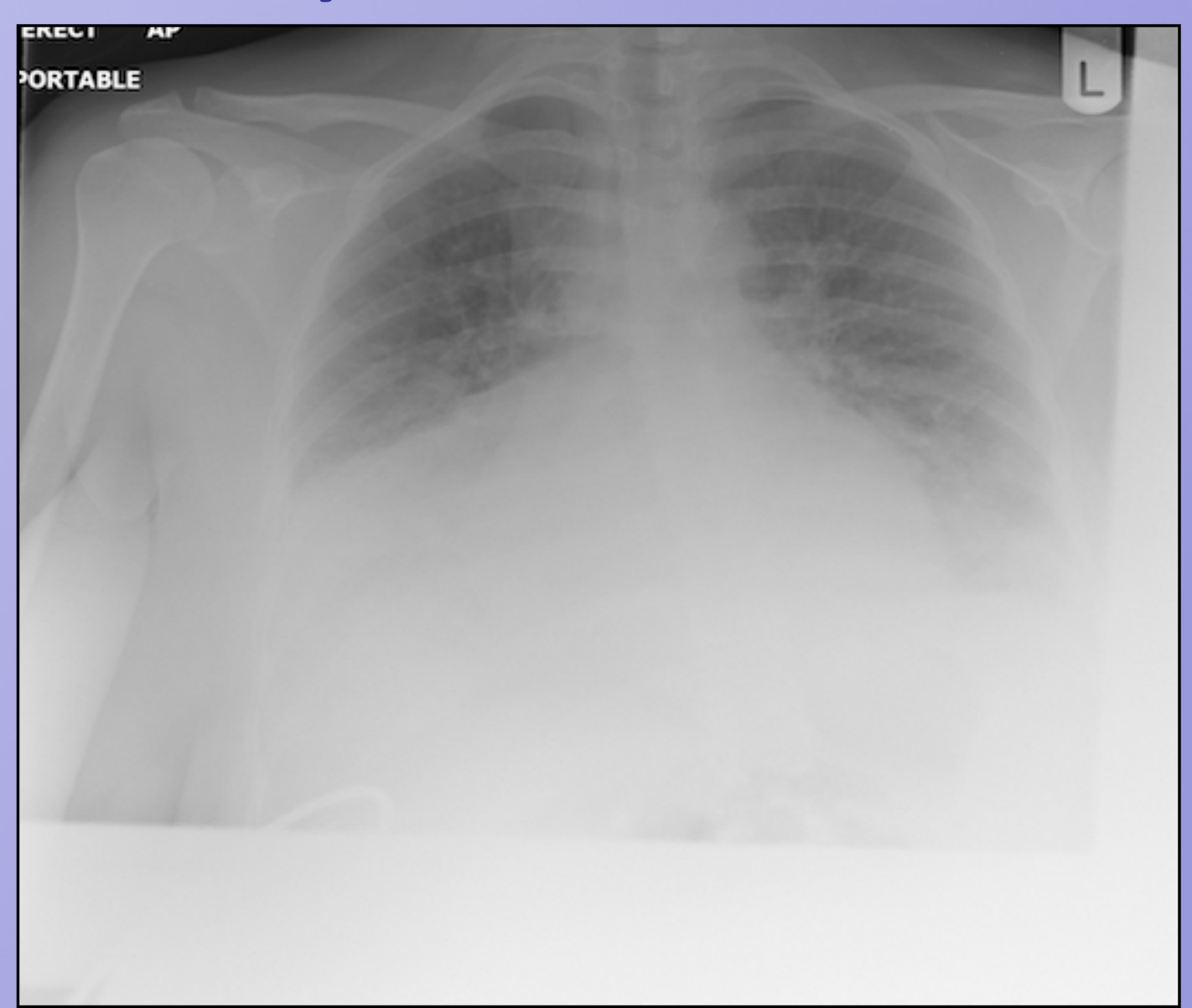

\section{Discussion}

o Pre-eclampsia, influenza infection, asthma, and respiratory depression from remifentanyl all contributed to rapid deterioration.

- Many studies have confirmed regional anaesthetic techniques reduce morbidity and mortality in emergency caesarean section.

o Regional anaesthesia when supplemented with additional respiratory support measures could be used to avoid general anaesthesia, improve outcomes and reduce need for prolonged ventilatory support.

\section{Learning Points}

- The role of non-invasive respiratory support (HFNC, CPAP) to facilitate safe regional anaesthetic techniques for emergency caesarean in patients with respiratory compromise should be considered. 